\title{
A Context-Specific Interface Model for Architectural Design in the Virtual Environment
}

\author{
Burcu Senyapili and Bülent Özgüç*
}

There is an ongoing debate on the success of architectural software in meeting the designers' wishes and in being familiar to the way designers design. One dominant belief is that as architectural software introduces a work environment closer to that of the paper-based techniques, the efficiency of the use of such software in the profession will increase. We argue that it is not the question of making the digital environment familiar to the paper-based, but designing interfaces through which the users will be able to customize the digital environment according to their wishes.

This study introduces a context-specific transformation model to convert a state in the 'user+need space' to a digital aid in the virtual design space. This model incorporates a customization scale menu (CSM) to act with the menu options of the architectural software. In this model, the menu options are customized through the selections made on the CSM by the user. These selections will determine the required level of interaction between the software and the user, thus customizing the digital environment according to the user's needs.

\section{Introduction}

\section{Aim}

Architectural design process is concerned with the creation and representation of spaces. Architects have been using the paper-based techniques to carry out this process until recent years. Then, with the introduction of the digital media [1] to architecture, they were given the option of using the digital work environment. This environment introduced the opportunity to create, manipulate and simulate the archirectural space digitally. However, the environment, although being efficient and fast especially in the representation part of the design process, is considered to be unfamiliar to the way architects create. Thus, in spite of the fact that more architects and architectural firms get involved with computers everyday, a large number of them use the digital environment for representation purposes rather than creation.

Architects have not asked for an alternative design environment. They have been using the paper-based techniques for a long time and even the ability to use these techniques has become an indispensable part of the profession. As the digital work environment was made available to the architects, they were impressed by the speed and ease provided by this environment especially for presentation. This has become one of the major reasons in the fast acceptance of the digital work environment to the profession. However, as the architectural software are developed by non-architects, the archirects are bound to express their wishes and

"Bilkent University, Faculty of Art, Design and Architecture, Department of Interior Architecture and Environmental Design, 06523 Bilkent. Ankara, Turkey. complaints only after the sofware is produced, not during the production. Richens states the fact that the creativity shifts from the architect to the ones who write the standards, the databases and the engines to operate them [2]. Therefore, shortly after the emergence of the digital media in architecture, architects chose to employ them mainly for representing and simulating what has already been created (where they were very efficient) rather than for creating (where they found the digital environment 'unfamiliar'). We intend to find out how architects can get a hold of the emerging possibilities of the digital media and control the development according to their wishes instead of leaving the development in the hands of other professions.

Architects complain about not being able to be as free as they are with paper-based techniques while using the architectural software and many long for the strikes of the soft pencil [3]. To overcome these complaints that the architectural software are 'unfamiliar' to the way architects design, one major tendency is to force the architectural softwate to offer a work environment similar to that of the paper-based techniques. This is tried to be achieved by features like using pens as input devices, offering sketchy looking line quality, allowing file exchange between various sofware, integrating large libraries and increasing the menu choices. But then the software expand in such a manner that both the usertrying to see the composition of two basic geometrical shapes and a second one making a lighting analysis of a space have to go through the same steps and have to input the same amount of data to perform their two very different tasks. As such, new complaints arise concerning the amount of time required to design [4], amount of time required to get used to the new additions and versions [5], amount of decisions to be given in the 
form of data even at the initial steps of design while using the architectural sofware.

In this study, we initially aim at showing that the potential of using the digital environment for creation in architectural design is more than the paper-based techniques. We argue that it is actually the paper-based techniques that serve more for representation than for creation in architectural design. As such, trying to make the way we use the architectural software similar to the way the paper-based techniques reduces the sofrware's potential for being used for creation.

We aim at increasing the efficiency and use of architectural space simulation software by decreasing the choices of the designer while using the sofware rather than increasing them. Maulsby observes that what users really want is more than an intelligent interface, it is an interface adapted to their own way of working. Because of the economies of scale, he states, nearly all systems have to be thought for the generic user [6] Within this framework, we ain at developing an interface system which will not be adapted to each user, but will allow each user adapt the software's menu options. In other words, the model is developed to allow the designer customize any architectural space simulation software according to the way he designs, so that he will not have to customize the way he designs according to the software

\section{Metbodology}

Within this framework, we discuss that architectural design is a modeling process and architectural communication is its representation. We compare the potentials of both the paper-based and the digital media in architectural design and communication. Based on this discussion, we assert that the digital media are 'familiar' to the essence of design, this essence being the mental design model. So, to benefit the most from the digital media, instead of trying to bring its potential down to the level of paper-based techniques, architectural design must be re-defined in relation to the digital potential.

We then argue that the problem faced when using the archirectural sofware is nor based on the lack of familiarity but rather on the lack of adequate interface design.

To form such an adequate interface, we initially re-define architectura means of communication in the Cartesian space of the digital environ. ment, freeing it from the domain of the paper-based techniques. As such, we obtain a space where we can determine the level of architectural communication which is applicable to the architectural software. Next, we have to allow the user to define the level within this space. But, instead of loading the user with such a burden, we form another Cartesian space to indicate the user's expectation from the architectural software. Consequentlv, our task of forming the interface model becomes a transformation of a given point in the user's space to the digital space. We define a transformation between the two spaces and then test the possible cases and discuss the relevant implications.

\section{The Concept of Modelling in Architectural and Design Communication}

\section{The Design Model in the Creation Process}

We perceive, comprehend, implement and communicate with the environment via forming mental models of that environment. These models store the information about the environment and this information is referred to for purposes like evaluation, change, comparison and communication
The design process, also, depends upon models loaded with various kinds of information (form, dimensions, relations, materials, colors, structure, etc. of space) about the design. The mental design model acquires three aspects. The first one is information processing [7]. The mental design model is a dynamic model, meaning that it is capable of updating itself if there happens to be a change in any of the data it contains

The second one is interactivity. The model allows the designer to implement, change and make associations with other models if necessary. Sumner et.al. groups design problem-solving as the construction of partial solutions on the understanding of the current goals and specifications and evaluation of these solutions according to various criteria and constraints [8]. This process requires the designer to constantly manipulate the mental design model and refine it by checking the aspects of the design versus each specification.

The third one of these aspects is time. Each architectural mass is based on a mental design model, i.e. it is the representation of a mental design model. However, there are two major differences berween the architectural product and its mental design model; the first one is the physical existence, the second is the factor of time [7].

Architecture can be defined in four dimensions. While the three of these make up the architectural volume, the fourth dimension, that of time, is concerned with the perception of the first three dynamically.

This latter dimension for any architectural building can be determined on a time coordinate that runs parallel to history and can be named as the actual time coordinate (atc). On this coordinate, the architectural space is perceived dynamically, and lives through a life span where it is faced with issues like detenioration, maintenance, changes of use, and restoration [9]. This life span occupies a definite time period on the act.

On the other hand, any design model created to carry knowledge about the future architectural building acquires two time coordinates. The first one is (again) the actual time coordinate displaying the time period when the design takes place and is generally prior to the life span of the building The second one is the virtual time coordinate offering virtual time periods for the design model to be tested, analyzed and revised, imitating the life span of the future building (Fig. 1) [10]. On this coordinate, not only the performance analyses of different design alternatives (thermal, structural acoustics, lighting, etc. analysis) and maintenance analyses (deterioration, resistance to fire, earthquake, etc.) can be executed, but revisions to can also be implemented based on the results [10].

\section{The Design Model in Representation and Communication}

Architectural design communication takes place between the architect and the engineer, the colleague, the customer, the critic, etc. during the process of design. During this communication they refer to the design model, or rather, to the representations of the design model. The designer seeks ways to communicate abour the design through various displays of the design model. We may group the techniques for developing and displaying the design model in two; paper-based and digital media. Within the framework of the aspects of the mental design model as discussed above, let us evaluate both media.

Paper-based Media: In the long history of architecture, the media used extensively to display the design model have been the paper-based (drawings and mock-ups) and the verbal. However, paper-based media can only represent the design model partially and statically. Because, be it any kind of drawing or mock-up, it reflects the state of the design model 


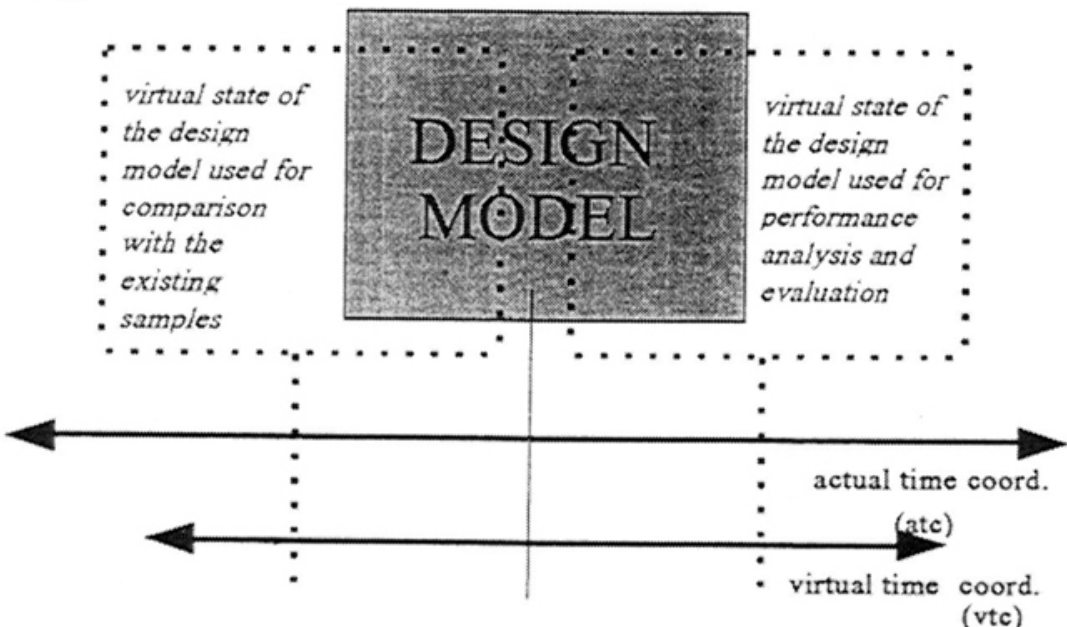

Figure 1. Actual and Virtual Time Coordinates of the Design Model
The digital media provide a menory which can hold one or more algorithms and the inpur data, and are capable of applying the algorithm to the data and displaying the result. The trivial form of this can be seen in the digital measuring devices, whereas the most improved case is the virtual reality en vironment.

As everything which is digitally coded is virually real, the territory of digital operations is also virtual, corresponding to a range on the vtc. This range on the vic forms the virtual work environment for the architect where the different states of the design model during various analyses can be sinulated (Fig. 2). The impressions gained from these vitual experiments that substitute the real ones $[13]$ result in various revisions. The representation of the design model as a digitized model then, turns out to be 'dynamic' which can continuously be updated similar to the mental design model.

at a certain point on the atc, and another on the vtc, the two points no corresponding to eachother. Such a representation refers to a certain time on the vtc, and the result is a static representation displaying the design model at that virtual moment, with limited amount of information relevant to that moment only.

Therefore, in the paper-based representations of architectural design there always occurs a difference, a gap between the design model and its representation [7]. The design model in the architect's mind is revised as he thinks, talks, and consults about the design. However, this revision cannot be easily applied to the design presented with the paper-based media, unlike the mental design model.

To illustrate this, let us imagine the exterior perspective drawing of a building. The drawing is completed at a certain date by the architect which denotes the actual time coordinate of the drawing. The drawing depicts the building at a certain hour (determines the angle and intensity of sunlight to be shed on the building facade) during a certain season which indicates its virtual time coordinate. As this virtual time coordinate consists of one point on the vtc, the information covered by this drawing is limited to that hour in that season and to the materials, colors and proportions shown on that drawing. Although the architect may decide to change the proportions of the windows, it will not be possible to show the revision until a new drawing is prepared. If there will be a question about the view while looking from inside to the ourside from one of the windows, the current perspective will not supply the answer, a new drawing will have to be made.

Digital Media: The digital media used in architecture include the digital distance measuring devices, stereophotogrammetry, optical digitizing, interactive movie map, 3D computer model [11] and - as everything that can be digitized can be simulated [12] all kinds of simulations made by the computer and by the digital camera.
To illustrate the dynamism of this digitized model, let us go back to our previous example and let us imagine the exterior perspective of the building on the screen of a computer with a high capacity. The perspective, however depicting the facade at a certain hour during a certain season, can quickly be altered to render the state of the same facade if the hour or the season or both were to be changed. Furthermore, changes, like the proportions of the windows, can be tested on the same drawing with ease. And, inquiries about another view taken from inside looking outside or the material properties of the surface cladding can be answered within a short period of time.

Based on the discussions above, we may point out that the paper-based media do not display the following three properties of the mental design model, whereas they are displayed by the digital media:
- dynamic perception of space
- performance analyses and
- instant adaptation

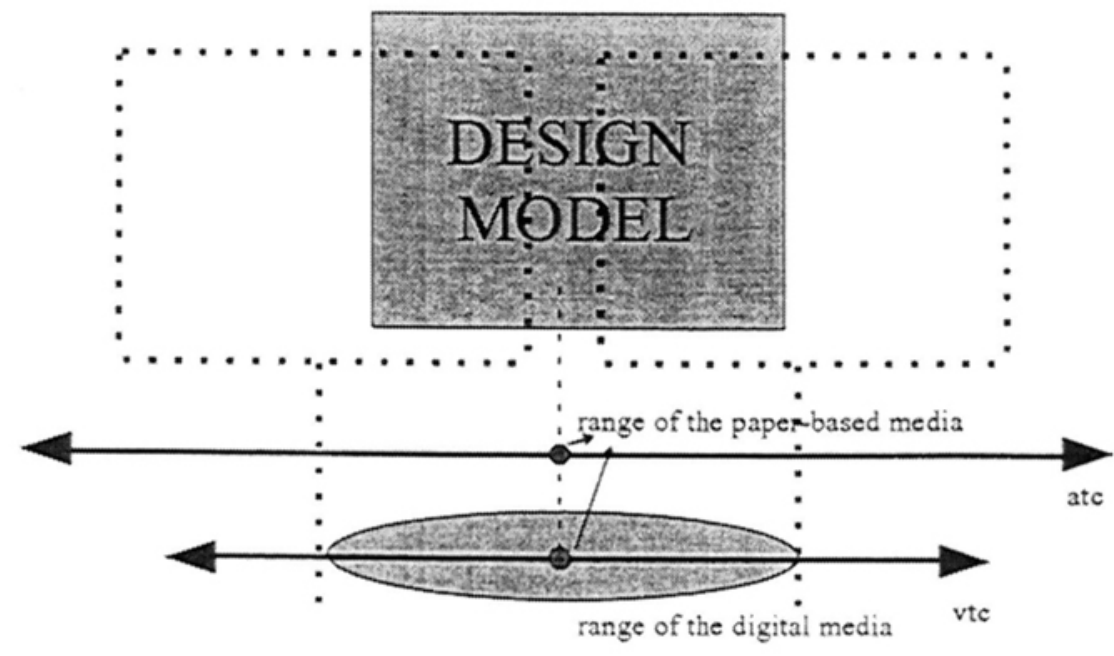

Figure 2. Ranges of Representation of the paper-based and the digital Media 


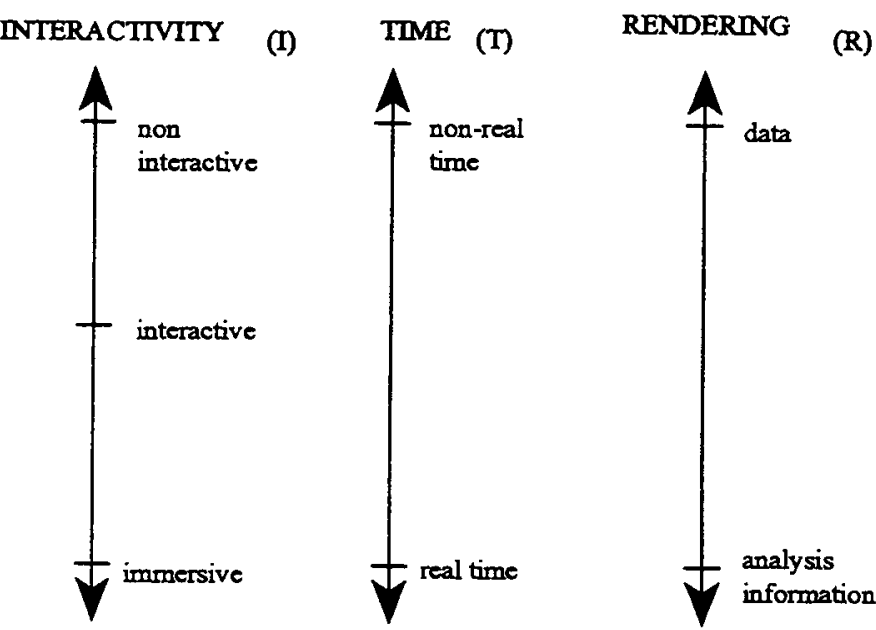

Figure 3. Scales of Heans of Architectural Communication

(R) rendering of the digitized model.

The next step is to test the scales to see whether they can be used to define all possible means of architectural design. Using the three scales mentioned above a 3D coordinate system is formed, at the center point of which paper-based media like drawings of plan, perspective, elevation, and sections can be placed [9]. If we construct a cube in this Cartesian space, each vertex can be used to address one of the means of architectural design communication (Fig. 4).

On the cube, the vertices stand for the following; 1 (paper-based drawings), 2 (verbal description), 3 (augmented wire-frame), 4 (video recording of a mock-up), 5 (walkthrough on video), 6 (photoreal still picture), 7 (3DCAD model), 8(the actual building itself). Different states of the architectural simulations in the virtual

These three properties depend on information processing, interactivity and dynamism in time. Although an architectural drawing made by the architect using pen and paper can be loaded aesthetically, it is very limited in providing design information, interactivity and dynamism [7].

The digitized model in the virtual environment transfers the mental design model to a medium where it can be shared and criticized by people other than the architect; where various analyses can be carried out and the results of both the critics and the analyses can be used to change or improve the design. The medium mentioned here is not the computer screen, but the virtual environment offered by the computer and other digital media [7].

It may then be argued that a well developed virtual environment is, in fact, a very familiar design environment. If designers have had the possibilities of such an environment instead of the paper-based techniques, today the discussions on the familiarity of the architectural software would not be based on their similarity to the paper-based techniques [14].

It is a difficult task to introduce a new design environment that requires different methods of handling, both in drafting and design, from those that the architects were accustomed to. Many architects will naturally long for the old techniques if they are faced with difficulties in understanding and manipulating the new environment and will complain that the newcomer is not familiar. Therefore, we need a transitional link, a flexible platform to enable the designers to get used to the new design environment by not adjusting the way they design according to the environment, but vice-versa.

\section{A Definition Model for Architectural Design in the Virtual Design Space}

To construct this transitional link, we need a model to define architectural design and communication within the boundaries of the virtual design environment. We have converted the above indicated factors of time, interactivity and information processing into the following three scales (Fig. 3). Information processing is determined as the level of environment then, can be defined on a line expanding from vertex 1 to vertex 8 [7].

This space gives the opportunity to address forms of architectural design communication within the dictionary of the virtual environment. We name this space as the virtual design space (VDS).

\section{The Space of User+Need}

The following step is to determine to which address the architect wants to go to in the VDS. To achieve this, we have to indicate the factors that make up that address, in other words, the factors in the user's domain that determine the context of simulation in the VDS. This context is unique to

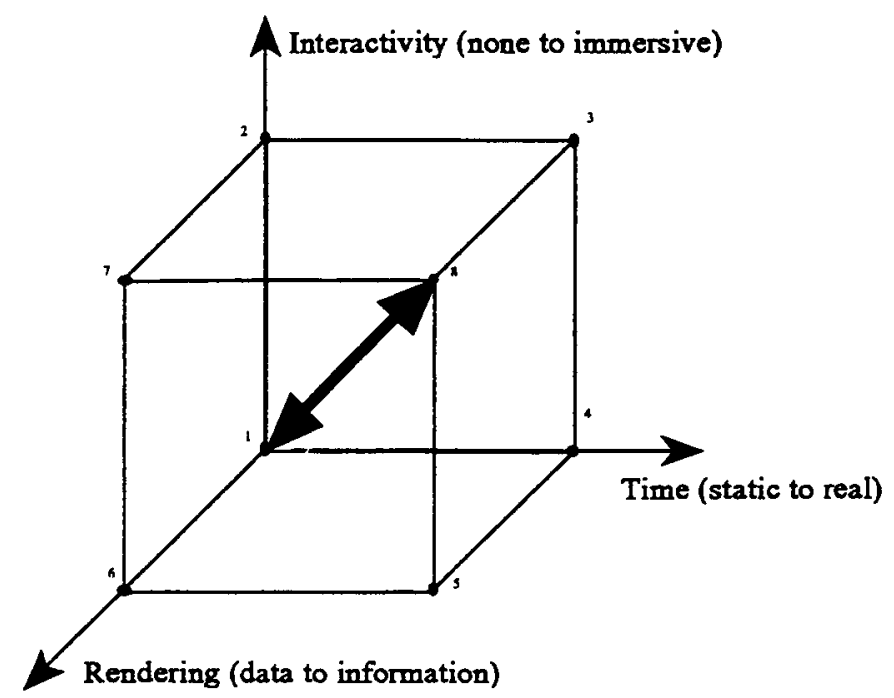

Figure 4. Arcbitecturual Communication as a Cube in 3D Coordinate System each user and to each performance, because the experience level of each user in using the software may differ as well as the purpose in using the software may differ for the same user at different times. The domain of the possible contexts is formed within the Cartesian space of user+need. This space is consisted of the following factors turned into coordinates.

\section{Purpose of Making the Simulation/ the Design Stage (P)}

The currently employed architectural space simulation software are 
onented in the same manner towards different kinds of needs, i.e. they introduce the same menu for different purposes, to users with different experience levels in using the sofware.

Architects complain about the amount of decisions to be given when making a simulation. The problem is not only the amount of time required for making all of these decisions, but the lack of the possibility in making of such precise decisions at the early stages of design. The complaint arises from the fact that the simulation software are programmed to serve in the same manner for different kinds of purposes. As a natural outcome, most of the architectural space simulations are made for the sake of having used the software and reveal little about the architectural quality and structure of the space. Then, it turns out to be a challenge as Emmett [15] points out, for the architects to go beyond the standard applications and reveal the qualities of the architectural design. Architects can get lost within the large range of menus that can lead them to miss the point in making the simulation. They can end up producing some simulation different from the one that was intended to [16]. Considerations in preparing an architectural space simulation are very closely related to those of moviemaking since both record motion. Nevertheless, it is not

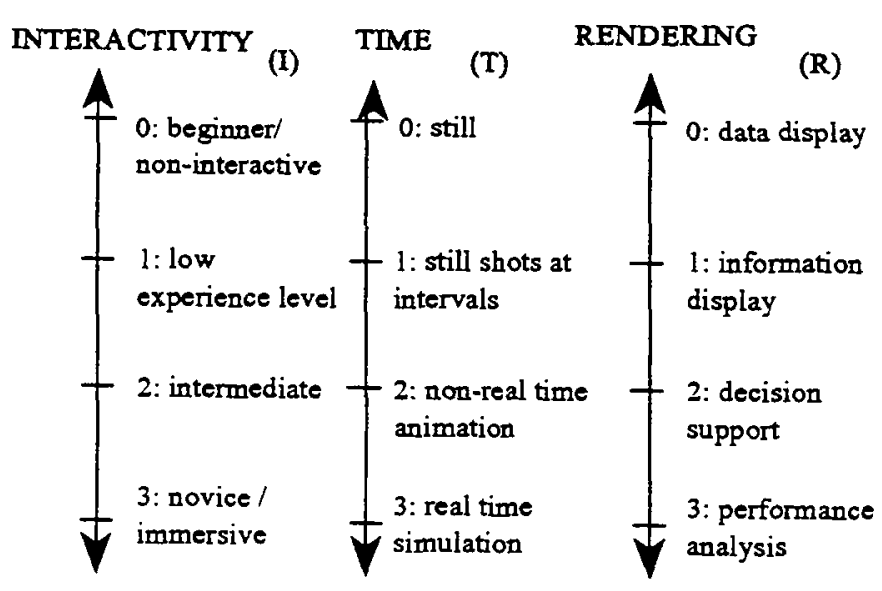

Figure 5. Scales of Means of Arcbitectural Communication

relevant to require architects to be educated in filmmaking just for the sake of making a successful simulation. It is the task of the simulation interface to aid the user with some defautr assignments and suggestions.

\section{Expectations of the Audience (A)}

The architectural space simulation is always made for an audience. In some cases the audience may be the architect him/herself, in others the audience may vary from a colleague, to a customer, an engineer or a design competition jury who all expect to see different aspects of the designed space. Whoever the audience is, architects try to communicate their projects so that the audience perceives it in the same way as the architect does. This is very crucial before the actual construction begins [17]

\section{Experience Level of the User in Using the Software (E)}

In spite of the recent additions and revisions to the currently used sofware, the problems of the "long learning cuive" [17] [4] and underdeveloped interfaces [18] still exist, requiring a long time and effort to learn, be able to manipulate and make the correct selection from the extensive menus. Contradicting the belief that this freedom can be obtained by increasing the menus and options, we choose to be on the camp of the ill effects of too many choices when using products of technology [19] and assert that the freedom can be maintained by downsizing the menus and the options.

\section{Customisation Scale Menu (CSM)}

Richens asserts that for a $C A D$ system to gain wide acceptance, it should be based visually and freed from cheory, rule and knowledge [2]. As such, the user, independent of the rules, theories and programming knowledge, should be able to indicate his/her aim in using the software and the software must be intelligent enough to customize itself according to the user's needs. Within this framework, the customization scale menu (CSM) is introduced to enable the user indicate his/her choices before using the architectural simulation software. The menu is responsible of running the program according to the indications given, thus narrowing down the choices and making some automatically.

CSM is the model of a transformation design which transforms a state in the PAE space (the space of user + need) to a digital aid in the IRT space (the virtual design space). The relationship of the PAE space with the IRT space is shown below.

$$
\begin{aligned}
& I=f_{1}(P, A, E) \\
& R=f_{R}(P, A, E) \\
& T=f_{T}(P, A, E)
\end{aligned}
$$

This relationship is basically the addressing of the equivalent in the I, R, T space of a point selected in the P, A, Espace. This can be explained with a transformation matix.

$$
\left[\begin{array}{l}
R \\
T
\end{array}\right]\left[\begin{array}{l}
P \\
A \\
E
\end{array}\right]\left[\begin{array}{ccc}
u_{I} & v_{I} & w_{I} \\
u_{R} & v_{R} & w_{R} \\
u_{T} & v_{T} & w_{T}
\end{array}\right]
$$

This transformation from the user's requirements to the virtual environment forms the context for the interface, indicating the state of the software to run at. This state is determined by the intervals on the three scales of $I, R$ and $T$ (Fig. 5).

The value of $I$ is the determinant of the default value assignments. If the addressed $I$ is between 0 and 1 , the default values will be assigned for various menu options; if between 1 and 2 , a suggested value will be displayed for the same menu options with the possibility of user's intervention and if between 2 and 3 , the user will be handling the assignment of the menu option.

The value of $R$ indicates the level of information. If $R$ is between 0 and 1 the display contains data only, made intentionally incapable of analyzing these data, if between 1 and 2 information is provided in relation to the database (shown as color and texture on the model), if between 2 and 3 the results of a performance analysis are rendered (shown as light on the model)

The value of $T$ determines the speed of the display. If $T$ is between 0 and 1 the display is still, if between 1 and 2 the display consists of snapshots in sequence and if between 2 and 3 the display is a motion picture [7].

Within this framework, each context determined by the $(I, R, T)$ set has a corresponding assignment (default, suggested value with the possibility of intervention or user's choice) for each menu option. The possible 2? different states of this set are shown below: 


\begin{tabular}{|c|c|c|}
\hline Default & Suggested & User choice \\
\hline$(\mathrm{I}, \mathrm{R}, \mathrm{T})$ & $(\mathrm{I}, \mathrm{R}, \mathrm{T})$ & $(\mathrm{I}, \mathrm{R}, \mathrm{D})$ \\
\hline$(0,0,0)$ & $(1,0,0)$ & $(2,0,0)$ \\
\hline$(0,0,1)$ & $(1,1,0)$ & $(2,1,0)$ \\
\hline$(0,0,2)$ & $(1,2,0)$ & $(2,2,0)$ \\
\hline$(0,1,0)$ & $(1,0,1)$ & $(2,0,1)$ \\
\hline$(0,2,0)$ & $(1,0,2)$ & $(2,0,2)$ \\
\hline$(0,1,1)$ & $(1,1,1)$ & $(2,1,1)$ \\
\hline$(0,2,1)$ & $(1,1,2)$ & $(2,1,2)$ \\
\hline$(0,1,2)$ & $(1,2,1)$ & $(2,2,1)$ \\
\hline$(0,2,2)$ & $(1,2,2)$ & $(2,2,2)$ \\
\hline
\end{tabular}

For instance, for the sub-menu item 'camera angle,' where $I=0$ will be the case that the camera angle will be determined as $\mathrm{x}$ degrees and this item will not be made available to the user. Where $I=1$, the angle will be set to $x$ degrees with the possibility of user's intervention. When $I=2$, the camera angle slot will appear empty for user's input.

Special states of CSM $(0,0,0),(1,1,1)$ and $(2,2,2)$ determine the extends of the software program. The state of $(0,0,0)$ indicates the use of the software as a paper-based tool, not different from any of the paper-based methods. The state of $(1,1,1)$ indicates the state at which many of the market available software presently run. Finally, the state $(2,2,2)$ indicates a level of immersion in the virtual environment where the user is in control of every facility. To us, the most important states are the intermediate ones, which cannot be realized alone without the CSM.

\section{Final Words: Expected Results}

The more relevant the context, bigher the value and longer the lifespan of the information [20].

CSM is a menu that indicates the level for the architectural space simulation software to run at, in other words, the context for the interface to operate. For instance, a student at the early stages of design may wish to operate at a very low interactive level since he/she is not capable of determining most of the values needed to form the simulation. At the low level of interaction, the required values for some of the menu options (e.g. illumination level, camera angles, camera moves etc.) will be automatically assigned based on the standard values. Since at the early stages of design, the same user may be satisfied to study the space in the form of a wire-frame model only. And finally, he/she may want to have a nonreal time simulation to be able to spend as much time as possible to comprehend the frames. Within the framework of these needs he/she is expected to select the early design stage as the purpose, him/herself as the audience and beginner as the experience level on the CSM. Based on these selections CSM assigns standard values for some menu options and highlights others for the user to assign values to and hides some of the menu options which are irrelevant at that purpose of simulation [9].

As such, the user with no prior experience of the application can make use of the default schemes and standard values of camera position, lighting level etc. An architect, at the initial phases of a design problem, may only give the global dimensions of the space and experience the design at different levels of lighting or color schemes. A design firm can display a highly rendered simulation to the client, while they show the same data in a longer and unrendered version to the construction engineers. Moreover, the design firm may produce a series of similar simulations for different designs, allowing comparisons in between [9].

There is one particular problem that needs close attention while working with the simulation software. The virtual environment is a model. It is indeed, a conglomeration of different models with different scaling factors. The physical building definition, on the other hand, has a dimensional scale, represented in meters, centimeters, feet, inches or even pixels. Rendering models lighting, surface textures, materials, etc. The scale of rendering is a difficulc one to define. If dimensional scale is an assumed ' $n$,' determining the corresponding rendering scale is even more difficult. In other words, if we are planning to use red marble in the real building, what color of which virtual material should a virtual nodel of dimensional scale ' $n$ ' show to model the scales correctly?

This problem is further complicated when orher scales, such as time, are superimposed. If, for example, it takes ten minutes to walk through the actual building, how long should it take to walkthrough a virtual building of dimensional scale ' $n$ '? Would this be in accordance with the rendering scale? There is actually not a known solution to this problem, known as true modeling. CSM can be an intuitive solution in mixing the different scales in one virtual modeling environment.

The optimization of CSM for the values between certain intervals is not an underestimation of the choices in the indicated intervals, rather it is an aid in using the virtual environment for creating successful displays. The provision of visual abstractions is more suitable to the designers' cognition, rather than the provision of almost the same perceptual experience of being inside the architectural space [21], which is not yet technically achieved. In this case, CSM can determine a level of optimized menu values, which is actually a very difficult task if left to the user.

CSM also helps the architects by reducing the menus and options. Since every state of the software determined by the user has a unique address in terms of $P, A, E$ and $I, R, T$ it is possible to a series of stylistically similar simulations for different designs [21]. By the use of such customization, architectural simulations may be brought down to a standard whenever needed. Such simulations lack of the means to define main circulation paths, cardinal views and important moves [22]. Since the contexts both in the user+need space and the VDS have addresses in terms of $(\mathrm{P}, \mathrm{A}, \mathrm{E})$ and $(I, R, T)$, they can be saved and be used again if necessary.

\section{References}

1 MITCHELL, W.J. and MCCULLOUGH, M., Digital Design . Hedia. New York: Van Nostrand Reinhold, 1991.

2 RICHENS, P., Does Knowledge Really Help? CAD Research at the Martin Centre, Knowledge-Based,Computer-Aided Arcbitectural Design, G. Carrara and Y.E. Kalay (Ed.s). London: Elsevier Science B.V., pp. 305-325, 1994.

3 O'CONNELL, K.R., Computer \& Architecture. School Arts, 94:9 16, 1995.

4 POTTER, C.D., Architectural CAD on the Desktop. Computer Graphics World, 19:6 25-35, 1995.

5 CHARLES, C. B. and BROWN, K.M.. Visualization Frontiers. Arcbitecture, $84: 12$ 121, 1995.

6 MAULSBY, D., Inductive Task Modeling for User Interface Customization. Intelligent User Interfaces IU1 97, J. Moore et.al. (Ed.s). New York: ACM, pp. 233-237, 1997.

7 SENYAPILI, Burcu. Visualization of Virtual Architecture. Information 
Visualization 97. California: IEEE, pp. 260-266, 1997

8 SUMNER, T. et.al., The Cognitive Ergonomics of Knowledge-Based Design Support Systems. Human Factors in Computing Systems, $\mathrm{S}$. Pemberton (Ed.). New York: ACM, pp. 83-91, 1997.

9 SENYAPILI, Burcu. Proposal to Improve the Use of Dynamic Simulations in Architectural Design. $4 D$ Dynamics, A. Robertson (Ed.). Leicester, DeMontfort University, pp. 151-155, 1995

10 SENYAPILI, Burcu. The True Model Concept in Computer Simulations Used in Architectural Design.6th Conference of the European FullScale .Modeling Association (EFA), B. Martens (Ed.)

Vienna: Vienna University of Technology Iris-Isis-Publications OKK Editions, pp. 133-140,1996.

11 BURDEN, E., Design Simulation. London: The Mitchell Publishing Company Limited, 1988.

12 BINKLEY, T., Digital Dilemmas. Leonardo (Digital Image-Digital Cinema Supplemental Issue), pp. 13-19, 1990.

13 MAHDAVI, A., Computational Decision Support and the Building Delivery Process: A Necessary Dialogue. Descriptive Models of Design, O. Akyn and G. Saglamer (Ed.s). Istanbul: Istanbul Technical University, 1996.
14 SENYAPILI, B. and OZGUÇ, B., Proposal tor Improving Computer Aid in Interior Architectural Design. Arcbitectural Science Review, $37: 4$ $171 \cdot 179,1994$

15 EMMETT, A., A New Dynamism. Computer Graphics World, 15:630 33, 1992.

16 MAHONEY, D.P., Walking Through Architectural Designs. Computer Graphics World, 17:6 22-30,1994

17 KEMPFER, L., Maximizing Visualization. Computer-aided Engineering, 15:6 48-54, 1996

18 SINGH, G., et.al., Virtual Reality: Software and Technology. Communications of the ACM, 39:535,1996.

19 NEGROPONTE, N., Being Digital. New York: Alfred A. Knopf, Inc., 1995.

20 SANDERS, K., The Digital Architect. New York:J. Wiley \& Sons, 1996

21 MARK, E., Case Studies in Moviemaking and Computer-Aided Design. The Electronic Design Studio,M. McCullough, W.J. Mitchell, and P. Purcell (Eds.). Massachusetts: The MIT Press, pp. 393- 412, 1990.

22 MARK, E., Architecture in Motion. Computers in Arcbitecture: Tools for Design, F. Penz (Ed.). London: Longman Group UK Ltd., pp. 9-16, 1992. 